\title{
基肥配比和拔节期追施氮肥对糯玉米淀粉胶凝和回生特性的影响
}

\author{
陆大雷 ${ }^{1}$ 王德成 ${ }^{1}$ 景立权 $^{1} \quad$ 韩 $^{\text {晴 }^{1}} \quad$ 郭换粉 $^{1} \quad$ 赵久然 $^{2}$ 陆卫平 ${ }^{1, *}$
}

${ }^{1}$ 扬州大学江苏省作物遗传生理重点实验室 / 农业部长江中下游作物生理生态与栽培重点开放实验室, 江苏扬州 $225009 ;{ }^{2}$ 北京市农 林科学院玉米研究中心, 北京 100097

摘 要: 以苏玉糯 4 号为材料, 采用二因素裂区设计, 研究了不同基肥配比(纯 $\mathrm{N} 75 \mathrm{~kg} \mathrm{hm}^{-2}$ 、纯 $\mathrm{N} 75 \mathrm{~kg} \mathrm{hm}^{-2}+\mathrm{K}_{2} \mathrm{O} 70$ $\mathrm{kg} \mathrm{hm}^{-2}$ 、纯 $\mathrm{N} 75 \mathrm{~kg} \mathrm{hm}^{-2}+\mathrm{P}_{2} \mathrm{O}_{5} 65 \mathrm{~kg} \mathrm{hm}^{-2}$ 和纯 $\left.\mathrm{N} 75 \mathrm{~kg} \mathrm{hm}^{-2}+\mathrm{P}_{2} \mathrm{O}_{5} 65 \mathrm{~kg} \mathrm{hm}^{-2}+\mathrm{K}_{2} \mathrm{O} 70 \mathrm{~kg} \mathrm{hm}^{-2}\right)$ 和拔节期追氮 $(0$ 、150 和 $300 \mathrm{~kg} \mathrm{hm}^{-2}$ ) 对糯玉米淀粉胶凝和回生特性的影响。结果表明, 淀粉和回生淀粉的起始温度、峰值温度和终值温度 虽然受到施肥处理的影响, 但总体上变异较小。热焓值受基肥配比影响较小, 糊化范围和峰值指数受拔节期追施氮肥 量影响较小。在不同基肥配比处理下，糊化范围在基施氮钾时最低，氮磷钾合理配施处理下最高，峰值指数表现和糊 化范围相反。在拔节期不同追氮量处理下, 热焓值以追氮 $150 \mathrm{~kg} \mathrm{hm}^{-2}$ 最高, 追氮 $300 \mathrm{~kg} \mathrm{hm}^{-2}$ 或不追氮无显著差异。 胶凝淀粉冷藏后发生回生, 表现为转变温度、热焓值和峰值指数降低, 糊化范围变宽。和仅基施氮相比, 增施磷或(和) 钾都可降低淀粉的回生值和回生淀粉的热焓值, 拔节期追氮处理的这两项指标均劣于不追氮处理。回生值与回生淀 粉的热焓值和峰值指数显著正相关, 相关系数分别为 $0.90(P<0.01)$ 和 $0.41(P<0.05)$; 原淀粉的热焓值与峰值指数极 显著正相关, 相关系数为 $0.65(P<0.01)$, 与回生淀粉热焓值显著正相关, 相关系数为 $0.44(P<0.05)$, 与终值温度显 著负相关, 相关系数为 $-0.41(P<0.05)$ 。在本试验条件下，以氮磷钾均衡基施并拔节期追氮 $150 \mathrm{~kg} \mathrm{hm}^{-2}$ 时, 淀粉胶凝 和回生特性较为理想, 表现为热焓值较高, 回生值较低。

关键词：糯玉米；淀粉; DSC；胶凝；回生；基肥配比；拔节期追氮

\section{Starch Gelatinization and Retrogradation Properties under Different Basic Fertilizer Regimes and Nitrogen Topdressing at Jointing Stage of Waxy Maize}

\author{
LU Da-Lei ${ }^{1}$, WANG De-Cheng ${ }^{1}$, JING Li-Quan ${ }^{1}$, HAN Qing ${ }^{1}$, GUO Huan-Fen ${ }^{1}$, ZHAO Jiu-Ran ${ }^{2}$, and \\ LU Wei-Ping ${ }^{1, *}$

\begin{abstract}
${ }^{1}$ Key Laboratory of Crop Genetics and Physiology of Jiangsu Province / Key Laboratory of Crop Physiology, Ecology and Cultivation in Middle and Lower Reaches of Yangtse River of Ministry of Agriculture, Yangzhou University, Yangzhou 225009, China; ${ }^{2}$ Maize Research Center, Beijing Academy of Agricultural and Forestry Sciences, Beijing 100097, China
\end{abstract}

\begin{abstract}
Proper fertilizer managements can improve the starch gelatinization and retrogradation properties of waxy maize (Zea mays L. Ceratina Kulesh). The split-design experiment was conducted using waxy maize cultivar Suyunuo 4 with four main plots subjected to basal fertilizer treatments of $\mathrm{N} 75 \mathrm{~kg} \mathrm{ha}^{-1}, \mathrm{~N} 75 \mathrm{~kg} \mathrm{ha}^{-1}+\mathrm{K}_{2} \mathrm{O} 70 \mathrm{~kg} \mathrm{ha}^{-1}, \mathrm{~N} 75 \mathrm{~kg} \mathrm{ha}^{-1}+\mathrm{P}_{2} \mathrm{O}_{5} 65 \mathrm{~kg} \mathrm{ha}^{-1}$, and N $75 \mathrm{~kg}$ $\mathrm{ha}^{-1}+\mathrm{P}_{2} \mathrm{O}_{5} 65 \mathrm{~kg} \mathrm{ha}^{-1}+\mathrm{K}_{2} \mathrm{O} 70 \mathrm{~kg} \mathrm{ha}^{-1}$, respectively in 2007 and 2008. The three split plots were topdressed with nitrogen of 0 , 150 , and $300 \mathrm{~kg} \mathrm{ha}^{-1}$ at jointing stage, respectively. Though onset temperature $\left(T_{\mathrm{o}}\right)$, peak temperature $\left(T_{\mathrm{p}}\right)$ and conclusion temperature $\left(T_{\mathrm{c}}\right)$ of native starch and retrogradated starch were affected by fertilizer treatments, the variation was little. No significant effects were observed for basic fertilizer treatments on the enthalpy of gelatinization $\left(\Delta H_{\text {gel }}\right)$, and for $\mathrm{N}$ topdressing treatments on gelatinization range $(R)$ and peak height index (PHI). The $R$-value was the lowest in the treatment with only $\mathrm{N}$ and $\mathrm{K}$ in basal fertilizer, and the highest in the treatment with $\mathrm{N}, \mathrm{P}$, and $\mathrm{K}$ in basal fertilizer. The changes of PHI showed an opposite trend to $R$. The $\Delta H_{\text {gel }}$ was the highest in the treatment with medium $\mathrm{N}$ topdressing $\left(150 \mathrm{~kg} \mathrm{ha}^{-1}\right)$. Gelatinized starch became retrograded with lower $T_{\mathrm{o}}, T_{\mathrm{p}}, T_{\mathrm{c}}$, enthalpy of retrogradation $\left(\Delta H_{\mathrm{ret}}\right)$, and $\mathrm{PHI}$, as well as wider $R$. Compared with the treatment of only $\mathrm{N}$ applied in basal fertilizer, the percentage of retrogradation and $\Delta H_{\text {ret }}$ decreased when $\mathrm{P}$ and/or $\mathrm{K}$ added in the basal fertilizer. The two indicators were higher in the treatments with $\mathrm{N}$ topdressing than without $\mathrm{N}$ topdressing. The percentage of retrogradation was posi-
\end{abstract}

\footnotetext{
本研究由国家自然科学基金项目(30270831)和北京市自然科学基金项目(YZPT02-06)资助。

“通讯作者(Corresponding author): 陆卫平, E-mail: wplu@yzu.edu.cn; Tel: 0514-87979377

第一作者联系方式: E-mail: ludalei0691@sina.com

Received(收稿日期): 2008-08-11; Accepted(接受日期): 2009-02-11.
} 
tively correlated with $\Delta H_{\text {ret }}(r=0.90, P<0.01)$ and PHI of retrograded starch $(r=0.41, P<0.05)$. $\Delta H_{\text {gel }}$ was positively correlated with PHI $(r=0.65, P<0.01)$ and $\Delta H_{\text {ret }}(r=0.44, P<0.05)$, but negatively correlated with $T_{\mathrm{c}}(r=-0.41, P<0.05)$. It is recommended to apply balanced fertilization with $\mathrm{N}, \mathrm{P}$, and $\mathrm{K}$ combining with moderate $\mathrm{N}$ topdressing under conditions similar to the experiment to improve gelatinization and retrogradation properties of waxy maize starch.

Keywords: Waxy maize; Starch; DSC; Gelatinization; Retrogradation; Basal fertilizer ratio; N topdressing at joint stage

胶凝和回生特性是淀粉重要的理化特性, 通常 利用差示扫描量热法(DSC)进行测定 ${ }^{[1]}$ 。评价淀粉胶 凝和回生特性的最主要特征参数是热焓值和回生值, 其中热焓值反映淀粉结构的稳定性, 而回生值主要 反映糊化淀粉在冷藏过程中的重结晶程度 ${ }^{[1]}$ 。对淀 粉胶凝和回生特性的理解是其在食品及相关工业应 用的基础。因此, 前人针对淀粉的胶凝和回生特性 进行了大量研究, 但他们主要集中于淀粉的基因型 差异 ${ }^{[2-6]}$, 而较少涉及施肥处理的影响 ${ }^{[7]}$ 。Noda 等 ${ }^{[7]}$ 在两个氮、磷、钾水平下研究了甘薯淀粉的胶凝特 性, 发现其主要受基因型影响, 施肥水平对其影响 较小。

糯玉米胚乳淀粉主要受 $w x$ 隐性基因控制, 由近 $100 \%$ 支链淀粉组成, 比普通玉米和甜玉米具有较高 的热焓值和转变温度, 且具其他优良理化特性, 在工 业上广泛应用, 如用作增稠剂、黏合剂、稳定剂等 等。虽然对不同玉米类型淀粉胶凝和回生特性 ${ }^{[8-9]}$ 及其 受变性处理的影响 ${ }^{[10-11]}$ 已有一些研究, 但未曾见到 栽培措施, 尤其是施肥处理对糯玉米淀粉品质影响 的报道, 而作物的品质特性与栽培措施有很大关 系。因此, 开展施肥处理对糯玉米淀粉理化特性影 响的研究对生产中调控糯玉米淀粉品质具有重要意 义, 对优质糯玉米淀粉的生产有直接的指导作用。 本课题组曾报道了不同施肥处理对淀粉糊化特性的 影响 ${ }^{[12]}$, 在此基础上, 进一步开展了不同基肥配比和 拔节期追氮处理对糯玉米淀粉胶凝和回生特性影响的 研究, 以期为糯玉米淀粉品质调控提供参考。

\section{1 材料与方法}

\section{1 材料与试验设计}

苏玉糯 4 号为江苏沿江地区农科所育成的深加 工兼用型品种 ${ }^{[13]}$, 籽粒产量接近普通玉米杂交种水 平, 生育期 $115 \mathrm{~d}$, 株高 $1.85 \mathrm{~m}, 2003$ 年通过国家审 定, 目前在江苏、浙江、上海等南方糯玉米主栽区 有较大种植面积。

试验于 2007 年和 2008 年在扬州大学实验农牧 场进行, 2007 年 3 月 30 日播种, 4 月 11 日移栽, 7 月 20 日收获, 2008 年 6 月 28 日播种, 10 月 10 日收获。
大田前茬空闲, 2007 年土壤含全氮 $0.88 \mathrm{~g} \mathrm{~kg}^{-1}$ 、速效 氮 $37.2 \mathrm{mg} \mathrm{kg}^{-1}$ 、速效磷 $20.1 \mathrm{mg} \mathrm{kg}^{-1}$ 、速效钾 $86.2 \mathrm{mg}$ $\mathrm{kg}^{-1} ; 2008$ 年土壤含全氮 $0.93 \mathrm{~g} \mathrm{~kg}^{-1}$ 、速效氮 $43.5 \mathrm{mg}$ $\mathrm{kg}^{-1}$ 、速效磷 $25.1 \mathrm{mg} \mathrm{kg}^{-1}$ 、速效钾 $79.4 \mathrm{mg} \mathrm{kg}^{-1}$ 。

采用裂区设计, 以不同基肥处理(B)为主区, 设 4 个水平, 分别是纯氮 $75 \mathrm{~kg} \mathrm{hm}^{-2}$ (B1)、纯氮 $75 \mathrm{~kg}$ $\mathrm{hm}^{-2}+\mathrm{K}_{2} \mathrm{O} 70 \mathrm{~kg} \mathrm{hm}^{-2}$ (B2)、纯氮 $75 \mathrm{~kg} \mathrm{hm}^{-2}+\mathrm{P}_{2} \mathrm{O}_{5}$ $65 \mathrm{~kg} \mathrm{hm}^{-2}$ (B3) 和纯氮 $75 \mathrm{~kg} \mathrm{hm}^{-2}+\mathrm{P}_{2} \mathrm{O}_{5} 65 \mathrm{~kg} \mathrm{hm}^{-2}$ $+\mathrm{K}_{2} \mathrm{O} 70 \mathrm{~kg} \mathrm{hm}^{-2}(\mathrm{~B} 4)$, 在移栽或播种前施用; 以拔 节期追施氮 $(\mathrm{D})$ 为副区，设纯氮 $0(\mathrm{D} 1) 、 150(\mathrm{D} 2)$ 和 $300 \mathrm{~kg} \mathrm{hm}^{-2}$ (D3) 3 个水平。不同基肥与追肥处理组 合, 2 次重复, 共 24 个小区, 小区面积 $24 \mathrm{~m}^{2}$, 种植 密度为 5.25 万株 $\mathrm{hm}^{-2}$ 。其他管理措施统一按常规 要求实施。

\section{2 籽粒淀粉分离及淀粉胶凝和回生特性测定}

参考本课题组前期报道的方法 ${ }^{[12]}$ 分离籽粒淀 粉。采用德国 NETZSCH 公司生产的差示扫描量热 仪(DSC 200F3 Maia), 在 Sandhu 等 ${ }^{[6]}$ 的方法基础上 略作修改, 用于测定淀粉胶凝和回生特性。称取 5 $\mathrm{mg}$ 淀粉, 加 $10 \mu \mathrm{L}$ 超纯水, 将样品铝盒密封后置于 $4^{\circ} \mathrm{C}$ 冰箱平衡过夜, 在测试前取出回温 $1 \mathrm{~h}$, 然后放 入 $\mathrm{DSC}$ 中测定, 以 $10^{\circ} \mathrm{C} \mathrm{min}-1$ 使铝盒温度由 $20^{\circ} \mathrm{C}$ 升 至 $100^{\circ} \mathrm{C}$, 以密封空白铝盒作参照。记录和计算起始 温度 $\left(T_{\mathrm{o}}\right)$ 、峰值温度 $\left(T_{\mathrm{p}}\right)$ 、终值温度 $\left(T_{\mathrm{c}}\right)$ 、热焓值 $\left(\Delta H_{\mathrm{gel}}\right)$, 糊化范围 $(R)$ 和峰值指数 $(\mathrm{PHI})$, 其中, $R=2\left(T_{\mathrm{p}}-T_{\mathrm{o}}\right)$; $\mathrm{PHI}=\Delta H_{\mathrm{gel}} /\left(T_{\mathrm{p}}-T_{\mathrm{o}}\right)$ 。

将分析样品于 $4^{\circ} \mathrm{C}$ 保存 $7 \mathrm{~d}$ 后进行淀粉的回生特

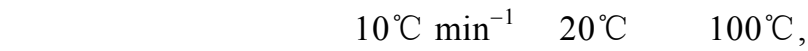
以密封空白铝盒作参照, 记录和计算相应参数。回 生值 $(\% R)$ 为回生淀粉的热焓值 $\left(\Delta H_{\mathrm{ret}}\right)$ 和原淀粉的热 焓值 $\left(\Delta H_{\mathrm{gel}}\right)$ 之比。

\section{3 数据分析}

采用 SAS 9.12 和 DPS 3.0 软件进行统计及相关 分析，采用 $L S D$ 法测验显著性。

\section{2 结果与分析}

2.1 不同施肥处理下糯玉米籽粒淀粉的胶凝特性 起始温度、峰值温度和终值温度虽然在两年间 
表现略有不同, 但总体上变异较小(3 个指标的变异 系数 2007 年分别为 $0.6 、 0.7$ 和 $0.8,2008$ 年分别为 $0.8 、 1.0$ 和 1.7), 说明施肥处理对其调控效应较小。 热焓值和峰值指数在年度间表现相同, 其中不同 基肥处理下拔节期追氮量对峰值指数影响较小; 随着追氮量的增加, 热焓值在 B3 和 B4 处理中呈先 升后降趋势, 在 B1 处理中呈先降后升趋势, 在 B2 处理中则表现为逐渐下降。2007 年糊化范围在不同 处理间无显著差异, 2008 年除 B1 处理外, 其他基
肥配比处理的表现与 2007 年相同。两年平均, B4D2 处理的淀粉热焓值最高 $\left(50.8 \mathrm{~J} \mathrm{~g}^{-1}\right)$ 、糊化范围较宽 (10.8)、峰值指数较低 $(9.5) ; \mathrm{B} 1 \mathrm{D} 3$ 和 B2D1 的淀粉 热焓值较高(分别 $49.3 \mathrm{~J} \mathrm{~g}^{-1}$ 和 $49.5 \mathrm{~J} \mathrm{~g}^{-1}$ )、糊化范围 较窄(均为 9.3)、峰值指数较高(分别为 10.7 和 10.9); B2D3 和 B4D1 处理中热焓值最低(均为 $45.5 \mathrm{~J} \mathrm{~g}^{-1}$ ), 但前者糊化范围和峰值指数均处于平均水平，而 后者糊化范围较宽(10.8), 峰值指数最低(仅为 8.6)。

表 1 不同施肥处理下糯玉米籽粒淀粉胶凝特征值

Table 1 Gelatinization characteristics of grain starch under different fertilizer treatments in waxy maize

\begin{tabular}{|c|c|c|c|c|c|c|}
\hline $\begin{array}{c}\text { 处理 } \\
\text { Treatment }\end{array}$ & $\begin{array}{c}\text { 起始温度 } \\
T_{\mathrm{o}}\left({ }^{\circ} \mathrm{C}\right) \\
\end{array}$ & $\begin{array}{c}\text { 峰值温度 } \\
T_{\mathrm{p}}\left({ }^{\circ} \mathrm{C}\right) \\
\end{array}$ & $\begin{array}{c}\text { 终值温度 } \\
T_{\mathrm{c}}\left({ }^{\circ} \mathrm{C}\right) \\
\end{array}$ & $\begin{array}{c}\text { 热焓值 } \\
\Delta H_{\mathrm{gel}}\left(\mathrm{J} \mathrm{g}^{-1}\right) \\
\end{array}$ & $\begin{array}{c}\text { 糊化范围 } \\
R \\
\end{array}$ & $\begin{array}{c}\text { 峰值指数 } \\
\text { PHI }\end{array}$ \\
\hline \multicolumn{7}{|l|}{2007} \\
\hline B1D1 & $68.1 \pm 0.9 \mathrm{~b}$ & $73.6 \pm 0.6 \mathrm{~b}$ & $82.2 \pm 0.7 \mathrm{ab}$ & $47.0 \pm 0.2 \mathrm{~cd}$ & $11.1 \pm 3.0 \mathrm{a}$ & $8.8 \pm 2.3 \mathrm{a}$ \\
\hline B1D2 & $68.7 \pm 0.4 \mathrm{ab}$ & $73.8 \pm 0.6 \mathrm{~b}$ & $80.9 \pm 0.4 \mathrm{~cd}$ & $43.0 \pm 1.0 \mathrm{~g}$ & $10.2 \pm 0.3 \mathrm{a}$ & $8.4 \pm 0.4 \mathrm{a}$ \\
\hline B1D3 & $69.4 \pm 0.4 \mathrm{a}$ & $74.2 \pm 0.6 \mathrm{~b}$ & $82.4 \pm 0.5 \mathrm{ab}$ & $47.7 \pm 0.0 \mathrm{bcd}$ & $9.7 \pm 0.4 \mathrm{a}$ & $9.8 \pm 0.4 \mathrm{a}$ \\
\hline B2D1 & $69.3 \pm 0.4 \mathrm{a}$ & $74.4 \pm 0.5 \mathrm{ab}$ & $82.1 \pm 0.3 \mathrm{ab}$ & $49.1 \pm 2.0 \mathrm{~b}$ & $10.1 \pm 1.8 \mathrm{a}$ & $9.9 \pm 1.4 \mathrm{a}$ \\
\hline B2D2 & $69.0 \pm 0.4 \mathrm{ab}$ & $74.4 \pm 0.4 \mathrm{ab}$ & $80.3 \pm 0.8 \mathrm{~d}$ & $47.6 \pm 0.7 \mathrm{bcd}$ & $10.7 \pm 1.6 \mathrm{a}$ & $9.0 \pm 1.2 \mathrm{a}$ \\
\hline B2D3 & $68.7 \pm 0.4 \mathrm{ab}$ & $73.9 \pm 0.6 \mathrm{~b}$ & $81.5 \pm 0.7 \mathrm{bc}$ & $44.4 \pm 0.9$ efg & $10.4 \pm 2.0 \mathrm{a}$ & $8.7 \pm 1.5 \mathrm{a}$ \\
\hline B3D1 & $68.2 \pm 0.3 \mathrm{ab}$ & $73.6 \pm 0.4 \mathrm{~b}$ & $81.6 \pm 0.3 \mathrm{abc}$ & $46.1 \pm 1.7 \mathrm{de}$ & $10.8 \pm 1.4 \mathrm{a}$ & $8.6 \pm 1.5 \mathrm{a}$ \\
\hline B3D2 & $68.5 \pm 0.5 \mathrm{ab}$ & $73.9 \pm 0.6 \mathrm{~b}$ & $82.1 \pm 0.3 \mathrm{ab}$ & $47.9 \pm 1.8 \mathrm{bc}$ & $10.8 \pm 2.3 \mathrm{a}$ & $9.1 \pm 2.2 \mathrm{a}$ \\
\hline B3D3 & $68.8 \pm 0.8 \mathrm{ab}$ & $73.8 \pm 0.6 \mathrm{~b}$ & $81.6 \pm 0.4 \mathrm{abc}$ & $45.2 \pm 0.8 \mathrm{ef}$ & $10.1 \pm 0.4 \mathrm{a}$ & $9.0 \pm 0.2 \mathrm{a}$ \\
\hline B4D1 & $69.3 \pm 0.4 \mathrm{a}$ & $75.3 \pm 0.6 \mathrm{a}$ & $82.4 \pm 0.1 \mathrm{ab}$ & $45.2 \pm 0.4 \mathrm{ef}$ & $12.1 \pm 1.8 \mathrm{a}$ & $7.6 \pm 1.2 \mathrm{a}$ \\
\hline B4D2 & $68.8 \pm 0.6 \mathrm{ab}$ & $74.3 \pm 0.4 \mathrm{ab}$ & $81.8 \pm 0.4 \mathrm{abc}$ & $51.5 \pm 0.2 \mathrm{a}$ & $11.1 \pm 0.4 \mathrm{a}$ & $9.3 \pm 0.3 \mathrm{a}$ \\
\hline B4D3 & $69.3 \pm 0.4 \mathrm{a}$ & $74.6 \pm 0.4 \mathrm{ab}$ & $82.5 \pm 0.6 \mathrm{a}$ & $44.1 \pm 1.7 \mathrm{fg}$ & $10.7 \pm 0.1 \mathrm{a}$ & $8.2 \pm 0.4 \mathrm{a}$ \\
\hline \multicolumn{7}{|l|}{2008} \\
\hline B1D1 & $68.9 \pm 0.6 \mathrm{~b}$ & $74.1 \pm 0.3 \mathrm{a}$ & $79.7 \pm 0.8 \mathrm{a}$ & $50.0 \pm 1.0 \mathrm{ab}$ & $10.4 \pm 0.6 \mathrm{ab}$ & $9.7 \pm 0.7 \mathrm{c}$ \\
\hline B1D2 & $68.6 \pm 0.1 \mathrm{bc}$ & $73.0 \pm 0.3 \mathrm{~cd}$ & $76.8 \pm 0.3 \mathrm{~cd}$ & $49.0 \pm 0.4 \mathrm{abc}$ & $8.8 \pm 0.4 \mathrm{~cd}$ & $11.2 \pm 0.5 \mathrm{abc}$ \\
\hline B1D3 & $69.8 \pm 0.4 \mathrm{a}$ & $74.2 \pm 0.1 \mathrm{a}$ & $78.0 \pm 0.1 \mathrm{abc}$ & $51.0 \pm 0.8 \mathrm{a}$ & $8.9 \pm 0.5 \mathrm{bcd}$ & $11.5 \pm 0.8 \mathrm{abc}$ \\
\hline B2D1 & $67.9 \pm 0.8 \mathrm{c}$ & $72.1 \pm 0.3 \mathrm{e}$ & $75.8 \pm 0.4 \mathrm{~d}$ & $49.8 \pm 0.1 \mathrm{ab}$ & $8.5 \pm 0.9 \mathrm{~cd}$ & $11.9 \pm 1.3 \mathrm{ab}$ \\
\hline B2D2 & $68.5 \pm 0.4 \mathrm{bc}$ & $72.4 \pm 0.1 \mathrm{de}$ & $76.4 \pm 0.3 \mathrm{~cd}$ & $48.4 \pm 0.7 \mathrm{abcd}$ & $7.8 \pm 0.6 \mathrm{~d}$ & $12.5 \pm 1.1 \mathrm{a}$ \\
\hline B2D3 & $68.3 \pm 0.2 \mathrm{bc}$ & $72.6 \pm 0.2 \mathrm{de}$ & $76.7 \pm 1.2 \mathrm{~cd}$ & $46.7 \pm 0.9 \mathrm{~cd}$ & $8.7 \pm 0.1 \mathrm{~cd}$ & $10.7 \pm 0.0 \mathrm{abc}$ \\
\hline B3D1 & $67.9 \pm 0.2 \mathrm{c}$ & $72.6 \pm 0.2 \mathrm{de}$ & $76.5 \pm 0.5 \mathrm{~cd}$ & $48.0 \pm 0.6 \mathrm{bcd}$ & $9.5 \pm 0.5 \mathrm{abc}$ & $10.2 \pm 0.7 \mathrm{bc}$ \\
\hline B3D2 & $69.0 \pm 0.8 \mathrm{ab}$ & $73.4 \pm 0.3 \mathrm{bc}$ & $77.7 \pm 0.8 \mathrm{bc}$ & $50.6 \pm 2.1 \mathrm{ab}$ & $8.8 \pm 1.0 \mathrm{~cd}$ & $11.7 \pm 1.7 \mathrm{abc}$ \\
\hline B3D3 & $68.7 \pm 0.6 \mathrm{~b}$ & $73.4 \pm 0.4 \mathrm{bc}$ & $78.7 \pm 0.3 \mathrm{ab}$ & $49.7 \pm 1.6 \mathrm{ab}$ & $9.4 \pm 0.4 \mathrm{abc}$ & $10.6 \pm 0.2 \mathrm{abc}$ \\
\hline B4D1 & $68.7 \pm 0.7 \mathrm{~b}$ & $73.4 \pm 0.4 \mathrm{bc}$ & $76.8 \pm 0.8 \mathrm{~cd}$ & $45.9 \pm 1.3 \mathrm{~d}$ & $9.5 \pm 0.5 \mathrm{abc}$ & $9.7 \pm 0.3 \mathrm{c}$ \\
\hline B4D2 & $68.9 \pm 0.9 \mathrm{~b}$ & $74.1 \pm 0.1 \mathrm{a}$ & $76.5 \pm 0.3 \mathrm{~cd}$ & $50.2 \pm 1.3 \mathrm{ab}$ & $10.5 \pm 1.5 \mathrm{a}$ & $9.8 \pm 1.6 \mathrm{bc}$ \\
\hline B4D3 & $69.0 \pm 0.8 \mathrm{ab}$ & $73.9 \pm 0.8 \mathrm{ab}$ & $79.7 \pm 0.3 \mathrm{a}$ & $48.0 \pm 1.6 \mathrm{bcd}$ & $9.8 \pm 0.0 \mathrm{abc}$ & $9.8 \pm 0.2 \mathrm{bc}$ \\
\hline
\end{tabular}

表中数据为 2 次重复的平均值土标准误。在同一年中, 每列数据后相同字母表示差异不显著 $(L S D$ 法)。第一列中, 字母 $\mathrm{B}$ 和 $\mathrm{D}$ 分 别代表基肥和拔节期追肥, B1 至 B4 施肥方案分别为 $\mathrm{N} 75 \mathrm{~kg} \mathrm{hm}^{-2} 、 \mathrm{~N} 75 \mathrm{~kg} \mathrm{hm}^{-2}+\mathrm{K}_{2} \mathrm{O} 70 \mathrm{~kg} \mathrm{hm}^{-2} 、 \mathrm{~N} 75 \mathrm{~kg} \mathrm{hm}^{-2}+\mathrm{P}_{2} \mathrm{O}_{5} 65 \mathrm{~kg} \mathrm{hm}^{-2}$ 和 N $75 \mathrm{~kg} \mathrm{hm}^{-2}+\mathrm{P}_{2} \mathrm{O}_{5} 65 \mathrm{~kg} \mathrm{hm}^{-2}+\mathrm{K}_{2} \mathrm{O} 70 \mathrm{~kg} \mathrm{hm}^{-2}$; $\mathrm{D} 1$ 至 $\mathrm{D} 3$ 表示追施纯氮 $0 、 150$ 和 $300 \mathrm{~kg} \mathrm{hm}^{-2}$ 。

Data in the table are mean $\pm S E$ of two replicates. In each year, values within a column followed by the same letter are not significantly different at $P<0.05$ according to $L S D$ test. In the first column, B and D refer to treatments of basal fertilizer and nitrogen topdressing, respectively. B1 to B4 denote treatments of $75 \mathrm{~kg} \mathrm{hm}^{-2}, \mathrm{~N} 75 \mathrm{~kg} \mathrm{hm}^{-2}+\mathrm{K}_{2} \mathrm{O} 70 \mathrm{~kg} \mathrm{hm}^{-2}, \mathrm{~N} 75 \mathrm{~kg} \mathrm{hm}^{-2}+\mathrm{P}_{2} \mathrm{O}_{5} 65 \mathrm{~kg} \mathrm{hm}^{-2}$, and N $75 \mathrm{~kg} \mathrm{hm}^{-2}+$ $\mathrm{P}_{2} \mathrm{O}_{5} 65 \mathrm{~kg} \mathrm{hm}^{-2}+\mathrm{K}_{2} \mathrm{O} 70 \mathrm{~kg} \mathrm{hm}^{-2}$, respectively. D1 to D3 denote treatments of nitrogen application of 0,150 , and $300 \mathrm{~kg} \mathrm{hm}^{-2}$, respectively. $T_{\mathrm{o}}$ : onset temperature; $T_{\mathrm{p}}$ : peak temperature; $T_{\mathrm{c}}$ : conclusion temperature; $\triangle H_{\mathrm{gel}}$ : enthalpy of gelatinization; $R$ : gelatinization range; PHI: peak height index. 
起始温度在基肥配比处理和追肥处理中比较稳定 且峰值温度在后者中比较稳定(表 2)。终值温度在两种 施肥方式处理中存在差异, 同时基肥配比处理对峰值温 度亦存在一定影响, 但总体上变幅较小 $\left(<1.2^{\circ} \mathrm{C}\right)$ 。在基肥
配比处理中, 热焓值相对稳定, 糊化范围在 B2 处理中 最低, 在 B4 处理下最高, 峰值指数的表现与糊化范围 相反。在追肥处理中, 糊化范围和峰值指数相对稳定, 热焓值在 D2 处理中最高, D1 或 D3 处理无显著差异。

表 2 不同施肥处理下糯玉米籽粒淀粉胶凝特征值的多重比较

Table 2 Multiple comparison of gelatinization characteristics of grain starch in different treatments in waxy maize

\begin{tabular}{|c|c|c|c|c|c|c|}
\hline $\begin{array}{c}\text { 处理 } \\
\text { Treatment }\end{array}$ & $\begin{array}{c}\text { 起始温度 } \\
T_{\mathrm{o}}\left({ }^{\circ} \mathrm{C}\right)\end{array}$ & $\begin{array}{c}\text { 峰值温度 } \\
T_{\mathrm{p}}\left({ }^{\circ} \mathrm{C}\right) \\
\end{array}$ & $\begin{array}{c}\text { 终值温度 } \\
T_{\mathrm{c}}\left({ }^{\circ} \mathrm{C}\right) \\
\end{array}$ & $\begin{array}{c}\text { 热焓值 } \\
\Delta H_{\mathrm{gel}}\left(\mathrm{J} \mathrm{g}^{-1}\right)\end{array}$ & $\begin{array}{c}\text { 糊化范围 } \\
R \\
\end{array}$ & $\begin{array}{c}\text { 峰值指数 } \\
\text { PHI }\end{array}$ \\
\hline \multicolumn{7}{|c|}{ 基肥配比 Basal fertilization } \\
\hline B1 & $68.9 \mathrm{a}$ & $73.8 \mathrm{~b}$ & $80.0 \mathrm{a}$ & $47.9 \mathrm{a}$ & $9.9 \mathrm{ab}$ & $9.9 \mathrm{ab}$ \\
\hline B2 & $68.6 \mathrm{a}$ & $73.3 \mathrm{c}$ & $78.8 \mathrm{~b}$ & $47.7 \mathrm{a}$ & $9.4 \mathrm{~b}$ & $10.4 \mathrm{a}$ \\
\hline B3 & $68.5 \mathrm{a}$ & $73.4 \mathrm{bc}$ & $79.7 \mathrm{a}$ & $47.9 \mathrm{a}$ & $9.9 \mathrm{ab}$ & $9.9 \mathrm{ab}$ \\
\hline B4 & $69.0 \mathrm{a}$ & $74.3 \mathrm{a}$ & 79.9 a & $47.5 \mathrm{a}$ & $10.6 \mathrm{a}$ & $9.1 \mathrm{~b}$ \\
\hline \multicolumn{7}{|c|}{ 氮肥追施 Nitrogen topdressing } \\
\hline D1 & $68.5 \mathrm{a}$ & $73.6 \mathrm{a}$ & $79.6 \mathrm{~b}$ & $47.6 \mathrm{~b}$ & $10.3 \mathrm{a}$ & $9.5 \mathrm{a}$ \\
\hline D2 & $68.7 \mathrm{a}$ & $73.7 \mathrm{a}$ & $79.1 \mathrm{c}$ & $48.5 \mathrm{a}$ & $9.8 \mathrm{a}$ & $10.1 \mathrm{a}$ \\
\hline D3 & $69.0 \mathrm{a}$ & $73.8 \mathrm{a}$ & $80.1 \mathrm{a}$ & $47.1 \mathrm{~b}$ & $9.7 \mathrm{a}$ & $9.8 \mathrm{a}$ \\
\hline
\end{tabular}

表中数据为所有处理小区的平均值。每列中数据后相同字母表示差异不显著 (LSD 法)。各处理的符号意义同表 1 。

Data in the table are means of all plots. Values followed by the same letter are not significantly different at $P<0.05$ according to LSD test. Treatments and abbreviations as in Table 1.

\section{2 不同施肥处理下糯玉米籽粒淀粉的回生特} 性

胶凝淀粉 $4{ }^{\circ} \mathrm{C}$ 冷藏 $7 \mathrm{~d}$ 后, 淀粉发生回生。和未 冷藏前相比，起始温度、峰值温度、终值温度、热 焓值和峰值指数显著降低, 糊化范围显著变宽(表 3)。回生淀粉的起始温度、峰值温度和终值温度在 年度间变化趋势有所不同, 但总体上变异程度均较 低(2007 年为 $2.2 、 0.7$ 和 $1.5,2008$ 年为 $2.2 、 2.5$ 和 4.7), 说明施肥处理对这 3 个指标调控效应较小。淀 粉的回生值在两年生长条件下表现相同, 即在 B1 和 B3 处理中随追肥量的增加而增加, 在 B2 处理中 随追肥量的增加呈先升后降趋势, 而在 B4 处理中 随追肥量的增加呈先降后升趋势。2007 年, 回生淀 粉的热焓值在 B1 和 $\mathrm{B} 2$ 处理中随追肥量的增加呈先 升后降趋势, 在 B3 和 B4 处理中分别呈增加和下降 趋势; 2008 年, 在 B1、B3 和 B4 处理中受追肥量的 影响较小, 在 B2 处理中则随追肥量的增加呈现先 升后降的趋势。糊化范围和峰值指数在两年种植条 件下略有不同(表 3), 但从两年平均值来看, 糊化范 围在 B1 处理中随氮肥追施量的增加呈先升后降趋势, 在 B4 处理中随氮肥追施量的增加呈先降后升趋势, 峰值指数在这两个处理中的变化和糊化范围相反; 在 B2、B3 处理中, 糊化范围表现为 D1、D2 下无显著差 异, 但高于 D3, 而峰值指数在 B2 处理中较为稳定, 在
B3 处理中则随氮肥追施量的增加而增加。回生值以 B4D2、B2D1 和 B3D1 处理中的较低(两年均值分别为 $51.1 \% 、 50.7 \%$ 和 $47.8 \%)$ ，以 B1D1、B1D2、B1D3、 $\mathrm{B} 3 \mathrm{D} 3$ 和 $\mathrm{B} 4 \mathrm{D} 1$ 处理较高(两年均值分别为 $60.1 \%$ 、 $63.0 \% 、 63.5 \%$ 、61.0\%和 $60.4 \%)$ 。

和 B1 相比, B2、 B3 和 B4 处理都降低了淀粉的 回生值和回生淀粉的热焓值, 说明增施磷或(和)钾 肥均可降低淀粉的回生，但 B4 和 B2、B3 处理间并 无显著差异, 说明施氮磷钾与施氮磷或氮、钾对其影 响较小(表 4)。和 D1 相比, D2、D3 处理均使其上升, 说明追氮对淀粉的回生值主要是负向作用。但从变 异系数来看, 回生淀粉的热焓值和回生值均表现为 基肥配比处理(分别为 6.6 和 6.3)高于追肥处理(分别 为 3.5 和 3.4), 说明基肥配施大于拔节肥对淀粉回生 的影响。起始温度、峰值温度和终值温度虽然受到 两种施肥方式的影响，但总体上变幅较小(表 4)。糊 化范围以 B2 处理最高, B3 和 B4 处理最低, 而在不 同追肥处理中以 $\mathrm{D} 3$ 处理最低。在不同基肥配比处 理中, 峰值指数以 $\mathrm{B} 1$ 处理最高、 $\mathrm{B} 2$ 处理最低, 且随 着氮肥追施量的增加而逐渐升高。

2.3 糯玉米籽粒淀粉胶凝特性和回生值之间的 相关分析

简单相关分析(表 5)表明，淀粉的回生值和回生 淀粉的热焓值和峰值指数显著正相关 (相关系数分 
表 3 不同施肥处理下糯玉米籽粒淀粉回生特征值

Table 3 Retrogradation characteristics of grain starch under different fertilizer treatments in waxy maize

\begin{tabular}{|c|c|c|c|c|c|c|c|}
\hline $\begin{array}{c}\text { 处理 } \\
\text { Treatment }\end{array}$ & $\begin{array}{c}\text { 起始温度 } \\
T_{\mathrm{o}}\left({ }^{\circ} \mathrm{C}\right) \\
\end{array}$ & $\begin{array}{c}\text { 峰值温度 } \\
T_{\mathrm{p}}\left({ }^{\circ} \mathrm{C}\right) \\
\end{array}$ & $\begin{array}{c}\text { 终值温度 } \\
T_{\mathrm{c}}\left({ }^{\circ} \mathrm{C}\right) \\
\end{array}$ & $\begin{array}{c}\text { 热焓值 } \\
\Delta H_{\text {ret }}\left(\mathrm{J} \mathrm{g}^{-1}\right)\end{array}$ & $\begin{array}{c}\text { 糊化范围 } \\
R \\
R\end{array}$ & $\begin{array}{c}\text { 峰值指数 } \\
\text { PHI }\end{array}$ & $\begin{array}{c}\text { 回生值 } \\
\% R\end{array}$ \\
\hline \multicolumn{8}{|l|}{2007} \\
\hline B1D1 & $46.5 \pm 0.4 \mathrm{abcd}$ & $55.5 \pm 0.5 \mathrm{ab}$ & $64.8 \pm 0.3 \mathrm{bcd}$ & $27.2 \pm 1.0 \mathrm{bc}$ & $17.9 \pm 1.8 \mathrm{~cd}$ & $3.0 \pm 0.2 \mathrm{bcd}$ & $57.9 \pm 1.9 \mathrm{bc}$ \\
\hline B1D2 & $46.7 \pm 0.4 \mathrm{abc}$ & $55.3 \pm 0.3 \mathrm{ab}$ & $64.2 \pm 0.4 \mathrm{de}$ & $26.8 \pm 0.8 \mathrm{bcd}$ & $17.3 \pm 0.1 \mathrm{cde}$ & $3.1 \pm 0.1 \mathrm{bc}$ & $62.4 \pm 0.4 \mathrm{ab}$ \\
\hline B1D3 & $46.4 \pm 0.6 \mathrm{bcd}$ & $54.9 \pm 0.2 \mathrm{bc}$ & $65.4 \pm 0.8 \mathrm{~b}$ & $30.1 \pm 1.3 \mathrm{a}$ & $17.0 \pm 0.8 \mathrm{cdef}$ & $3.5 \pm 0.3 \mathrm{a}$ & $63.2 \pm 2.6 \mathrm{a}$ \\
\hline B2D1 & $45.5 \pm 0.1 \mathrm{de}$ & $54.3 \pm 0.1 \mathrm{c}$ & $62.9 \pm 0.1 \mathrm{f}$ & $24.7 \pm 1.0 \mathrm{~cd}$ & $17.6 \pm 0.0 \mathrm{cde}$ & $2.8 \pm 0.1 \mathrm{bcd}$ & $50.3 \pm 0.0 \mathrm{~d}$ \\
\hline B2D2 & $44.4 \pm 0.6 \mathrm{f}$ & $54.7 \pm 0.3 \mathrm{bc}$ & $64.5 \pm 0.2 \mathrm{cde}$ & $26.9 \pm 0.2 \mathrm{bcd}$ & $20.6 \pm 1.7 \mathrm{ab}$ & $2.6 \pm 0.2 \mathrm{de}$ & $56.5 \pm 0.4 \mathrm{c}$ \\
\hline B2D3 & $47.1 \pm 0.7 \mathrm{ab}$ & $55.5 \pm 0.4 \mathrm{ab}$ & $65.1 \pm 0.3 \mathrm{bc}$ & $21.8 \pm 0.7 \mathrm{e}$ & $16.7 \pm 0.7 \mathrm{def}$ & $2.6 \pm 0.0 \mathrm{de}$ & $49.1 \pm 0.6 \mathrm{~d}$ \\
\hline B3D1 & $46.5 \pm 0.4 \mathrm{abcd}$ & $55.8 \pm 0.5 \mathrm{a}$ & $66.8 \pm 0.3 \mathrm{a}$ & $18.3 \pm 0.2 \mathrm{f}$ & $18.6 \pm 0.3 \mathrm{~cd}$ & $2.0 \pm 0.0 \mathrm{f}$ & $39.7 \pm 1.8 \mathrm{e}$ \\
\hline B3D2 & $45.8 \pm 0.6 \mathrm{cde}$ & $55.3 \pm 0.4 \mathrm{ab}$ & $64.5 \pm 0.3 \mathrm{cde}$ & $26.8 \pm 2.2 \mathrm{bcd}$ & $18.9 \pm 0.4 \mathrm{bc}$ & $2.8 \pm 0.3 \mathrm{bcd}$ & $56.0 \pm 6.6 \mathrm{c}$ \\
\hline B3D3 & $47.4 \pm 0.1 \mathrm{a}$ & $55.0 \pm 0.5 \mathrm{bc}$ & $64.6 \pm 0.1 \mathrm{~cd}$ & $27.5 \pm 1.5 \mathrm{~b}$ & $15.1 \pm 0.7 \mathrm{f}$ & $3.6 \pm 0.4 \mathrm{a}$ & $60.8 \pm 4.5 \mathrm{abc}$ \\
\hline B4D1 & $45.1 \pm 0.1$ ef & $55.5 \pm 0.1 \mathrm{ab}$ & $65.1 \pm 0.2 \mathrm{bc}$ & $27.7 \pm 1.3 \mathrm{ab}$ & $20.7 \pm 0.1 \mathrm{ab}$ & $2.7 \pm 0.1 \mathrm{cde}$ & $61.3 \pm 3.5 \mathrm{ab}$ \\
\hline B4D2 & $46.9 \pm 0.2 \mathrm{ab}$ & $54.8 \pm 0.4 \mathrm{bc}$ & $63.8 \pm 0.3 \mathrm{e}$ & $25.6 \pm 1.6 \mathrm{bcd}$ & $15.9 \pm 0.4 \mathrm{ef}$ & $3.2 \pm 0.3 \mathrm{ab}$ & $49.7 \pm 3.3 \mathrm{~d}$ \\
\hline B4D3 & $44.2 \pm 0.6 \mathrm{f}$ & $55.0 \pm 0.3 \mathrm{abc}$ & $65.1 \pm 0.6 \mathrm{bc}$ & $24.7 \pm 0.7 \mathrm{~d}$ & $21.6 \pm 0.6 \mathrm{a}$ & $2.3 \pm 0.1 \mathrm{ef}$ & $56.0 \pm 0.6 \mathrm{c}$ \\
\hline \multicolumn{8}{|l|}{2008} \\
\hline B1D1 & $44.7 \pm 0.5 \mathrm{~cd}$ & $55.4 \pm 0.6 \mathrm{~d}$ & $67.5 \pm 0.4 \mathrm{de}$ & $31.2 \pm 0.3 \mathrm{a}$ & $21.4 \pm 2.2 \mathrm{def}$ & $3.0 \pm 0.3 \mathrm{ab}$ & $62.3 \pm 0.4 \mathrm{ab}$ \\
\hline B1D2 & $44.4 \pm 0.4 \mathrm{~cd}$ & $57.5 \pm 0.6 \mathrm{bc}$ & $72.9 \pm 0.2 \mathrm{ab}$ & $31.1 \pm 0.1 \mathrm{a}$ & $26.2 \pm 2.0 \mathrm{bc}$ & $2.4 \pm 0.2 \mathrm{cde}$ & $63.5 \pm 0.6 \mathrm{a}$ \\
\hline B1D3 & $44.9 \pm 0.2 \mathrm{bcd}$ & $56.3 \pm 0.5 \mathrm{~cd}$ & $70.8 \pm 0.6 \mathrm{c}$ & $32.5 \pm 0.7 \mathrm{a}$ & $22.8 \pm 0.6 \mathrm{cde}$ & $2.9 \pm 0.2 \mathrm{abc}$ & $63.8 \pm 0.7 \mathrm{a}$ \\
\hline B2D1 & $43.7 \pm 0.6 \mathrm{~d}$ & $59.6 \pm 0.2 \mathrm{a}$ & $74.6 \pm 0.4 \mathrm{a}$ & $25.5 \pm 0.6 \mathrm{~d}$ & $31.8 \pm 0.8 \mathrm{a}$ & $1.6 \pm 0.0 \mathrm{f}$ & $51.2 \pm 1.2 \mathrm{e}$ \\
\hline B2D2 & $44.1 \pm 0.1 \mathrm{~cd}$ & $58.4 \pm 0.2 \mathrm{ab}$ & $73.7 \pm 0.4 \mathrm{ab}$ & $28.9 \pm 1.0 \mathrm{bc}$ & $28.6 \pm 0.6 \mathrm{ab}$ & $2.0 \pm 0.1 \mathrm{ef}$ & $59.6 \pm 1.4 \mathrm{~b}$ \\
\hline B2D3 & $46.4 \pm 0.4 \mathrm{ab}$ & $58.6 \pm 0.3 \mathrm{ab}$ & $68.6 \pm 0.2 \mathrm{~d}$ & $27.4 \pm 0.6 \mathrm{~cd}$ & $24.4 \pm 1.4 \mathrm{~cd}$ & $2.3 \pm 0.1 \mathrm{de}$ & $58.8 \pm 2.2 \mathrm{bc}$ \\
\hline B3D1 & $45.3 \pm 0.5 \mathrm{abc}$ & $55.7 \pm 0.3 \mathrm{~d}$ & $72.7 \pm 0.3 \mathrm{~b}$ & $26.9 \pm 0.8 \mathrm{ab}$ & $20.8 \pm 0.4 \mathrm{def}$ & $2.6 \pm 0.1 \mathrm{bcd}$ & $56.0 \pm 2.1 \mathrm{~cd}$ \\
\hline B3D2 & $45.2 \pm 0.5 \mathrm{abcd}$ & $56.1 \pm 0.4 \mathrm{~cd}$ & $66.7 \pm 1.3 \mathrm{ef}$ & $30.5 \pm 0.8 \mathrm{ab}$ & $21.8 \pm 0.2 \mathrm{def}$ & $2.8 \pm 0.1 \mathrm{abc}$ & $60.3 \pm 0.3 \mathrm{ab}$ \\
\hline B3D3 & $46.6 \pm 0.7 \mathrm{a}$ & $56.1 \pm 0.9 \mathrm{~cd}$ & $67.1 \pm 0.9 \mathrm{def}$ & $30.5 \pm 0.6 \mathrm{ab}$ & $19.0 \pm 0.4 \mathrm{ef}$ & $3.2 \pm 0.0 \mathrm{a}$ & $61.3 \pm 0.3 \mathrm{ab}$ \\
\hline B4D1 & $45.5 \pm 0.8 \mathrm{abc}$ & $55.1 \pm 0.3 \mathrm{~d}$ & $66.2 \pm 0.0$ ef & $27.3 \pm 0.8 \mathrm{~cd}$ & $19.2 \pm 2.2$ ef & $2.9 \pm 0.3 \mathrm{abc}$ & $59.5 \pm 0.6 \mathrm{bc}$ \\
\hline B4D2 & $46.7 \pm 0.2 \mathrm{a}$ & $55.7 \pm 0.1 \mathrm{~d}$ & $65.6 \pm 0.1 \mathrm{f}$ & $26.3 \pm 0.8 \mathrm{~d}$ & $18.0 \pm 0.2 \mathrm{f}$ & $2.9 \pm 0.1 \mathrm{abc}$ & $52.5 \pm 2.5 \mathrm{de}$ \\
\hline B4D3 & $46.3 \pm 0.5 \mathrm{ab}$ & $56.2 \pm 0.8 \mathrm{~cd}$ & $67.5 \pm 0.5 \mathrm{de}$ & $26.4 \pm 0.8 \mathrm{~d}$ & $19.8 \pm 0.6 \mathrm{ef}$ & $2.7 \pm 0.0 \mathrm{bcd}$ & $55.0 \pm 0.2 \mathrm{~d}$ \\
\hline
\end{tabular}

表中数据为 2 次重复的平均值土标准误。在同一年中，每列数据后相同字母表示差异不显著( $L S D$ 法)。

Data in the table are mean $\pm S E$ of two replicates. In each year, values within a column followed by the same letter are not significantly different at $P<0.05$ according to $L S D$ test. Treatments and abbreviations as in Table $1 . \% R$ : the percentage of retrogradation.

表 4 不同施肥处理下糯玉米籽粒淀粉回生特征值的多重比较

Table 4 Multiple comparison of retrogradation characteristics of grain starch under different fertilizer treatments in waxy maize

\begin{tabular}{|c|c|c|c|c|c|c|c|}
\hline $\begin{array}{c}\text { 处理 } \\
\text { Treatment }\end{array}$ & $\begin{array}{c}\text { 起始温度 } \\
T_{\mathrm{o}}\left({ }^{\circ} \mathrm{C}\right) \\
\end{array}$ & $\begin{array}{c}\text { 峰值温度 } \\
T_{\mathrm{p}}\left({ }^{\circ} \mathrm{C}\right) \\
\end{array}$ & $\begin{array}{c}\text { 终值温度 } \\
T_{\mathrm{c}}\left({ }^{\circ} \mathrm{C}\right) \\
\end{array}$ & $\begin{array}{c}\text { 热焓值 } \\
\Delta H_{\mathrm{gel}}\left(\mathrm{J} \mathrm{g}^{-1}\right) \\
\end{array}$ & $\begin{array}{c}\text { 糊化范围 } \\
R \\
R \\
\end{array}$ & $\begin{array}{c}\text { 峰值指数 } \\
\text { PHI }\end{array}$ & $\begin{array}{c}\text { 回生值 } \\
\% R\end{array}$ \\
\hline 基肥配比 & Basal fertilization & & & & & & \\
\hline B1 & $45.6 \mathrm{bc}$ & $55.8 \mathrm{~b}$ & $67.6 \mathrm{~b}$ & $29.8 \mathrm{a}$ & $20.4 \mathrm{~b}$ & $3.0 \mathrm{a}$ & $62.2 \mathrm{a}$ \\
\hline B2 & $45.2 \mathrm{c}$ & $56.8 \mathrm{a}$ & $68.2 \mathrm{a}$ & $25.8 \mathrm{c}$ & $23.3 \mathrm{a}$ & $2.3 \mathrm{c}$ & $54.2 \mathrm{~b}$ \\
\hline B3 & $46.1 \mathrm{a}$ & $55.6 \mathrm{~b}$ & $67.1 \mathrm{~b}$ & $26.7 \mathrm{~b}$ & $19.0 \mathrm{c}$ & $2.8 \mathrm{~b}$ & $55.7 \mathrm{~b}$ \\
\hline B4 & $45.8 \mathrm{ab}$ & $55.4 \mathrm{~b}$ & $65.5 \mathrm{c}$ & $26.3 \mathrm{bc}$ & $19.2 \mathrm{c}$ & $2.8 \mathrm{~b}$ & $55.6 \mathrm{~b}$ \\
\hline 氮肥追施 & Nitrogen topdressing & & & & & & \\
\hline D1 & $45.3 \mathrm{~b}$ & $55.8 \mathrm{a}$ & $67.6 \mathrm{a}$ & $26.1 \mathrm{~b}$ & $21.0 \mathrm{a}$ & $2.6 \mathrm{c}$ & $54.8 \mathrm{~b}$ \\
\hline D2 & $45.5 \mathrm{~b}$ & $56.0 \mathrm{a}$ & $67.0 \mathrm{~b}$ & $27.8 \mathrm{a}$ & $20.9 \mathrm{a}$ & $2.7 \mathrm{~b}$ & $57.5 \mathrm{a}$ \\
\hline D3 & $46.2 \mathrm{a}$ & $55.9 \mathrm{a}$ & $66.8 \mathrm{~b}$ & $27.6 \mathrm{a}$ & $19.6 \mathrm{~b}$ & $2.9 \mathrm{a}$ & $58.5 \mathrm{a}$ \\
\hline
\end{tabular}

表中数据为所有处理小区的平均值。每列中数据后相同字母表示差异不显著 (LSD 法)。各处理的符号意义同表 1 。

Data in the table are means of all plots. Values followed by the same letter are not significantly different at $P<0.05$ according to $L S D$ test. Treatments and abbreviations as in Tables 1 and 2 . 
别为 0.90 和 0.41 )。原淀粉的热焓值和峰值指数及回 生淀粉的热焓值显著正相关(相关系数分别为 0.65 和 0.44), 和终值温度显著负相关 (相关系数为 $-0.41)$ 。回生淀粉的热焓值与原淀粉的峰值指数显著 正相关(相关系数为 0.50 ), 和糊化范围显著负相关 (相关系数为 -0.41 )。胶凝特征参数中, 淀粉的峰值 温度和起始温度及终值温度极显著正相关(相关系 数分别为 0.76 和 0.74$) 。$ 糊化范围和峰值指数极显著 负相关(相关系数为 -0.92 ), 且前者和峰值温度及终 值温度极显著正相关(相关系数分别为 0.78 和 0.77 ), 后者和峰值温度及终值温度极显著负相关 $($ 相关系 数分别为 -0.70 和 -0.78$)$ 。回生特征参数中, 终值温 度和起始温度极显著负相关 (相关系数为 -0.57$)$, 和 峰值温度极显著正相关(相关系数为 0.82 )。糊化范 围和峰值指数极显著负相关 (相关系数为 -0.78 ), 前
者与起始温度极显著负相关 (相关系数为 -0.80$)$, 和 峰值温度和终值温度极显著正相关(相关系数分别 为 0.87 和 0.85 ), 后者与起始温度极显著正相关(相 关系数为 0.61$)$, 和峰值温度和终值温度极显著负相 关(相关系数分别为 -0.69 和 -0.61$)$ 。原淀粉的起始温 度、峰值温度和终值温度和回生淀粉的峰值温度、 终值温度显著负相关(相关系数分别为 $-0.48 、-0.78$ 、 -0.70 和 $-0.41 、-0.76 、-0.78)$, 和回生淀粉的峰值指数 显著正相关(相关系数分别为 $0.44 、 0.49$ 和 0.42 )。原 淀粉的峰值温度、终值温度和回生淀粉的糊化范围呈 显著负相关(相关系数分别为 -0.62 和 -0.67 )。原淀粉的 糊化范围和回生淀粉的峰值温度、终值温度、热焓值 和糊化范围显著负相关(相关系数分别为 $-0.71 、-0.75$ 、 -0.41 和 -0.66$)$, 然而峰值指数则表现为显著正相关 (相关系数分别为 $0.70 、 0.76 、 0.50$ 和 0.69 )。

表 5 籽粒淀粉胶凝和回生特征值之间的相关系数

Table 5 Correlation coefficients between retrogradation value and DSC characteristics of gelatinization of grain starch

\begin{tabular}{|c|c|c|c|c|c|c|c|c|c|c|c|c|c|}
\hline & & \multirow{2}{*}{$\% R$} & \multicolumn{6}{|c|}{ 胶凝特征值 Gelatinization characteristic } & \multicolumn{5}{|c|}{ 回生特征值 Retrogradation characteristic } \\
\hline & & & $T_{\mathrm{o}}$ & $T_{\mathrm{p}}$ & $T_{\mathrm{c}}$ & $\Delta H_{\mathrm{gel}}$ & $R$ & PHI & $T_{\mathrm{o}}$ & $T_{\mathrm{p}}$ & $T_{\mathrm{c}}$ & $\Delta H_{\text {ret }}$ & $R$ \\
\hline \multirow{6}{*}{$\begin{array}{c}\text { 胶凝特征值 } \\
\text { Gelatinization } \\
\text { characteristic }\end{array}$} & $T_{\mathrm{o}}$ & 0.34 & & & & & & & & & & & \\
\hline & $T_{\mathrm{p}}$ & 0.01 & $0.76^{* *}$ & & & & & & & & & & \\
\hline & $T_{\mathrm{c}}$ & -0.19 & 0.37 & $0.74^{* *}$ & & & & & & & & & \\
\hline & $\Delta H_{\mathrm{gel}}$ & 0.02 & 0.07 & -0.17 & $-0.41^{*}$ & & & & & & & & \\
\hline & $R$ & -0.29 & 0.19 & $0.78^{* *}$ & $0.77^{* *}$ & -0.34 & & & & & & & \\
\hline & PHI & 0.23 & -0.15 & $-0.70^{* *}$ & $-0.78^{* *}$ & $0.65^{* *}$ & $-0.92^{* *}$ & & & & & & \\
\hline \multirow{6}{*}{$\begin{array}{c}\text { 回生特征值 } \\
\text { Retrogradation } \\
\text { characteristic }\end{array}$} & $T_{\mathrm{o}}$ & -0.21 & -0.05 & 0.20 & 0.38 & -0.24 & 0.35 & $-0.43^{*}$ & & & & & \\
\hline & $T_{\mathrm{p}}$ & 0.06 & $-0.48^{*}$ & $-0.78^{* *}$ & $-0.70^{* *}$ & 0.22 & $-0.71^{* *}$ & $0.70^{* *}$ & -0.39 & & & & \\
\hline & $T_{\mathrm{c}}$ & 0.15 & $-0.41^{*}$ & $-0.76^{* *}$ & $-0.78^{* *}$ & 0.33 & $-0.75^{* *}$ & $0.76^{* *}$ & $-0.57^{* *}$ & $0.82^{* *}$ & & & \\
\hline & $\Delta H_{\text {ret }}$ & $0.90^{* *}$ & 0.34 & -0.06 & -0.35 & $0.44^{*}$ & $-0.41^{*}$ & $0.50^{*}$ & -0.29 & 0.15 & 0.29 & & \\
\hline & $R$ & 0.15 & -0.29 & $-0.62^{* *}$ & $-0.67^{* *}$ & 0.27 & $-0.66^{* *}$ & $0.69^{* *}$ & $-0.80^{* *}$ & $0.87^{* *}$ & $0.85^{* *}$ & 0.26 & \\
\hline & PHI & $0.41^{*}$ & $0.44^{*}$ & $0.49^{*}$ & $0.42^{*}$ & 0.02 & 0.34 & -0.33 & $0.61^{* *}$ & $-0.69^{* *}$ & $-0.61^{* *}$ & 0.37 & $-0.78^{* *}$ \\
\hline
\end{tabular}

${ }^{*}$ Significant at $P<0.05 .{ }^{* *}$ Significant at $P<0.01$. Abbreviations as in Tables 1 and 2.

\section{3 讨论}

Noda 等 ${ }^{[7]}$ 研究甘薯淀粉的理化特性时发现, 淀 粉的糊化和胶凝特性主要受基因型的影响, 而肥料 水平对其影响较小。糯玉米淀粉的糊化特性受到基 肥配比和拔节期追氮量的影响, 且糊化特征值在基 施氮磷钾并拔节期追氮 $150 \mathrm{~kg} \mathrm{hm}^{-2}$ 时较优 ${ }^{[12]}$ 。本研 究结果同样证实基肥配比和拔节期追氮对淀粉的胶 凝和回生特性存在显著影响, 在基施氮磷钾并拔节 期追氮 $150 \mathrm{~kg} \mathrm{hm}^{-2}$ 时淀粉具有较高的热焓值和较低 的回生值。可见, 通过合理的施肥运筹可以改良糯
玉米淀粉的理化特性。在本研究中, 基肥配比对热 焓值无显著影响，拔节期追氮 $150 \mathrm{~kg} \mathrm{hm}^{-2}$ 时较高, 不追氮或追氮 $300 \mathrm{~kg} \mathrm{hm}^{-2}$ 时较低，表明拔节期过量 追氮或不追氮会使淀粉结构的稳定性降低。

淀粉低温冷却后，糊化淀粉重新结晶，形成较 为松散的淀粉结构 ${ }^{[14]}$ ，因此起始温度、峰值温度、 终值温度、热焓值和峰值指数显著降低, 糊化范围 显著变宽。Singh 等 ${ }^{[1]}$ 统计不同来源淀粉回生值和转 变温度(起始温度至终值温度)时发现, 淀粉的回生 值为 $5 \% \sim 62 \%$, 转变温度较原淀粉低 $10 \sim 26^{\circ} \mathrm{C}$, 本研 究结果表明, 糯玉米不同处理下淀粉的回生值为 
$39.7 \% \sim 63.8 \%$ ，转变温度较原淀粉低 $12.5 \sim 23.0^{\circ} \mathrm{C}$, 且不同施肥处理间存在较大差异, 说明施肥处理对 淀粉的回生起着重要作用。从本文结果可知, 和仅 施氮作基肥相比, 增施磷或(和)钾可降低淀粉的回 生值, 拔节期追氮 $150 \mathrm{~kg} \mathrm{hm}^{-2}$ 或 $300 \mathrm{~kg} \mathrm{hm}^{-2}$ 对回生 值无显著影响, 但均高于不追氮处理, 说明拔节期 追氮对淀粉的回生主要起负向作用。对回生淀粉而 言, 其热焓值可定量评价重结晶支链淀粉熔化所需 的能量, 并可精确测量吸热过程中的转变温度 ${ }^{[15]}$, 峰值指数可用来评估胶凝的均匀性 ${ }^{[16]}$ 。本试验表明, 回生淀粉的热焓值和回生值基本一致, 且这两项指 标呈显著正相关, 较好地解释了回生值的变化是由 回生淀粉的热焓值所决定。从两年数据来看, 以基 施氮、钾或氮磷并拔节期不追氮和氮、磷钾均衡基施并 拔节期追氮 $150 \mathrm{~kg} \mathrm{hm}^{-2}$ 时回生值较低。结合淀粉胶 凝特性和前期 ${ }^{[12]}$ 关于淀粉糊化特性在不同处理下的 变化, 可认为在本试验相似条件下, 基施氮磷钾结 合拔节期追氮 $150 \mathrm{~kg} \mathrm{hm}^{-2}$ 有利于改善淀粉的胶凝特 性和回生特性。

回生值和胶凝特征值之间无显著相关关系, 和 回生淀粉的热焓值及峰值指数显著正相关。由于当 淀粉具有较高的热焓值时其淀粉的结晶度较高 ${ }^{[1]}$, 表明胶凝淀粉冷藏后重结晶程度越高, 结晶均匀性 越好, 淀粉越易回生。Xu 等 ${ }^{[17]}$ 在水稻上的研究亦同 样证实, 回生淀粉热焓值越高时, 淀粉的回生值越 高。在本研究中, 原淀粉的热焓值和峰值指数极显 著正相关, 说明淀粉的稳定性越高, 其胶凝糊化后 的均匀性越好; 峰值温度与起始温度和终值温度极显 著正相关, 这一结果与在普通玉米 ${ }^{[3,10,18]}$ 和水稻 ${ }^{[17,19]}$ 上的研究结果相似。但本研究发现, 淀粉的热焓值 和终值温度显著负相关, 和起始温度及峰值温度相 关不显著, 而在普通玉米 ${ }^{[3,10,18]}$ 和水稻 ${ }^{[17,19]}$ 上, 热焓 值和转变温度显著正相关。由于普通玉米和水稻淀 粉由直链淀粉和支链淀粉组成, 而直链淀粉对热焓 值存在显著影响 (普通玉米上为显著正相关 ${ }^{[10]}$, 水 稻上为显著负相关 ${ }^{[17]}$ ), 糯玉米淀粉几乎全部由支 链淀粉组成, 淀粉成分的不同可能是造成这种差异 的原因。同样, 淀粉的结晶度、颗粒大小和形态、 支链淀粉链长分配等均影响着淀粉的理化特性, 糯 玉米和其他类型玉米淀粉在这些指标上均存在着差

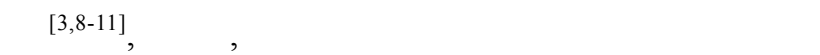
究将可能更好地为糯玉米淀粉品质调控服务。

\section{4 结论}

不同施肥处理引起淀粉胶凝和回生特性的变 化。热焓值和回生值在不同施肥处理下的变化趋势 相同, 其他参数略有差异。淀粉和回生淀粉的转变 温度(起始温度、峰值温度和终值温度)虽然受到施 肥处理的影响, 但总体上变异幅度较小, 施肥对其 调控效应较小。淀粉的热焓值受基肥配比影响较小, 在拔节期追氮 $150 \mathrm{~kg} \mathrm{hm}^{-2}$ 时最高, 追氮 $300 \mathrm{~kg} \mathrm{hm}^{-2}$ 或不追氮处理下无显著差异。与仅基施氮肥相比, 增施磷或(和)钾都可降低淀粉的回生值和回生淀粉 的热焓值, 但施氮磷钾和施氮磷或氮钾无显著差异, 且追氮对这两个指标呈负向影响。在本试验条件下, 不同施肥处理中以平衡基施氮磷钾并拔节期追氮 $150 \mathrm{~kg} \mathrm{hm}^{-2}$ 淀粉胶凝特性和回生特性较优, 即淀粉 结构较为稳定且回生值较低。

\section{References}

[1] Singh N, Singh J, Kaur L, Sodhi N S, Gill B S. Morphological, thermal and rheological properties of starches from different botanical sources. Food Chem, 2003, 81: 219-231

[2] Kuakpetoon D, Wang Y. Structural characteristics and physicochemical properties of oxidized corn starches varying in amylose content. Carbohyd Res, 2006, 341: 1896-1915

[3] Ji Y, Wong K, Hasjim J, Pollak L M, Duvick S, Jane J. Structure and function of starch from advanced generations of new corn lines. Carbohyd Polym, 2003, 5: 305-319

[4] Seetharaman K, Tziotis A, Borras F, White P J, Ferrer M, Robutti J. Thermal and functional characterization of starch from Argentinean corn. Cereal Chem, 2001, 78: 379-386

[5] Yamin F F, Lee M, Pollak L M, White P J. Thermal properties of starch in corn variants isolated after chemical mutagenesis of inbred line B73. Cereal Chem, 1999, 76: 175-181

[6] Sandhu S K, Singh N. Some properties of corn starches: II. Physicochemical, gelatinization, retrogradation, pasting and gel textural properties. Food Chem, 2007, 101: 1499-1507

[7] Noda T, Takahata Y, Sato T, Ikoma H, Mochida H. Physicochemical properties of starches from purple and orange fleshed sweet potato roots at two levels of fertilizer. Starch/Stärke, 2006, 48: 395-399

[8] Singh M, Sandhu K S, Kaur M. Physicochemical properties including granular morphology, amylose content, swelling and solubility, thermal and pasting properties of starches from normal, waxy, high amylose and sugary corn. Prog Food Biopolymer Res, 2005, 1: 43-54

[9] Singh N, Inouchi N, Nishinari K. Structural, thermal and viscoe- 
lastic characteristics of starches separated from normal, sugary and waxy maize. Food Hydrocolloid, 2006, 20: 923-935

[10] Sandhu K S, Singh N, Lim S. A comparison of native and acid thinned normal and waxy corn starches: Physicochemical, thermal, morphological and pasting properties. LWT Food Sci Technol, 2007, 40: 1527-1536

[11] Sandhu K S, Kaur M, Singh N, Lim S. A comparison of native and oxidized normal and waxy corn starches: physicochemical, thermal, morphological and pasting properties. LWT Food Sci Technol, 2008, 41: 1000-1010

[12] Lu D-L(陆大雷), Lu W-P(陆卫平), Zhao J-R(赵久然), Wang D-C(王德成). Effects of basic fertilizer treatments and nitrogen topdressing at jointing stage on starch RVA characteristics of waxy maize. Acta Agron Sin (作物学报), 2007, 34(7): 1253-1258 (in Chinese with English abstract)

[13] Lu H-H(陆虎华), Chen G-Q(陈国清)，Xue L(薛林)，Huang X-L(黄小兰), Shi M-L(石明亮), Yin Z-T(印志同). Study on the breeding of new waxy maize hybrid Suyunuo No.4 of fresh and processing type. J Jinling Inst Tech (金陵科技学院院报), 2005,
21(3): 58-61 (in Chinese with English abstract)

[14] Sasaki T, Yasui T, Matsuki J. Effect of amylase content on gelatinization, retrogradation and pasting properties of starches from waxy and non-waxy wheat and their $\mathrm{F}_{1}$ seeds. Cereal Chem, 2000, 77: $58-63$

[15] Karim A A, Norziah M H, Seow C C. Methods for the study of starch retrogradation. Food Chem, 2000, 71: 9-36

[16] Aggarwal V, Singh N, Kamboj S S, Brar P S. Some properties of seeds and starches separated from different Indian pea cultivars. Food Chem, 2004, 85: 585-590

[17] Xu L J, Xie J K, Kong X L, Bao J S. Analysis of genotypic and environmental effects on rice starch: 2 . Thermal and retrogradation properties. J Agric Food Chem, 2004, 52: 6017-6022

[18] Sandhu K S, Singh N, Malhi N S. Physicochemical and thermal properties of starches separated from corn produced from crosses of two germ pools. Food Chem, 2005, 89: 541-548

[19] Singh N, Kaur L, Sandhu K S, Kaur J, Nishinari K. Relationships between physicochemical, morphological, thermal, rheological properties of rice starches. Food Hydrocolloid, 2006, 20: 532-542 